\title{
Nucleon Structure Functions in a Chiral Soliton Model
}

\author{
Herbert Weigel ${ }^{\mathrm{a}} \mathrm{\oplus}$ \\ ${ }^{a}$ Center for Theoretical Physics \\ Laboratory of Nuclear Science and Department of Physics \\ Massachusetts Institute of Technology, Cambridge, Ma 02139, USA
}

The computation of nucleon structure functions within the Nambu-Jona-Lasinio chiral soliton model is outlined. After some technical remarks on the issue of regularization numerical results for the both unpolarized and polarized structure functions are presented. The generalization to flavor $\mathrm{SU}(3)$ is sketched.

\section{THE CHIRAL SOLITON MODEL}

The bosonized form of the Nambu-Jona-Lasinio (NJL) action [1]

$$
\mathcal{A}[S, P]=-i N_{C} \operatorname{Tr}_{\Lambda} \log \left[i \not \partial-\left(S+i \gamma_{5} P\right)\right]-\frac{1}{4 G} \int d^{4} x \operatorname{tr}\left[S^{2}+P^{2}+2 \hat{m}_{0}(S+i P)\right],
$$

represents the starting point for considering nucleon structure functions in a chiral soliton model. The action $\mathcal{A}$ is a functional of respectively scalar and pseudoscalar fields $S$ and $P$ which are matrices in flavor space. The VEV,$\langle S\rangle=m$ is obtained from the gap-equation and measures the dynamical breaking of chiral symmetry. For apparent reasons $m$ is called the constituent quark mass (matrix). The regularization of the quadratically divergent quark loop is indicated by the cut-off $\Lambda$. Its value as well as the coupling constant $G$ and the current quark mass $\hat{m}_{0}$ are adjusted to the phenomenological meson parameters $m_{\pi}$ and $f_{\pi}$, leaving only a single free parameter, commonly chosen to be $m$. The static soliton is constructed from the hedgehog ansatz on the chiral circle

$S+i \gamma_{5} P=m \exp \left(i \vec{\tau} \cdot \hat{r} \gamma_{5} \Theta(r)\right)=: m U_{5}$.

This defines a Dirac Hamiltonian $h=\vec{\alpha} \cdot \vec{p}+m \beta U_{5}$ with eigenvalues $\epsilon_{\alpha}$. The latter parameterize the regularized energy functional extracted from the action (1)

$E[\Theta]=N_{C}\left(\epsilon_{\mathrm{val}}-\frac{1}{2} \sum_{\alpha \Lambda}\left|\epsilon_{\alpha}\right|\right)$.

Here the subscript "val" refers to the distinct valence quark level which is strongly bound in the background of the hedgehog. The soliton is finally constructed by extremizing the

*Talk presented at the KEK-Tanashi Int. Symposium on the Physics of Hadrons and Nuclei, Tokyo, Dec. 1998, to appear in the proceedings. This work is supported in parts by funds provided by the U.S. Department of Energy (D.O.E.) under cooperative research agreement \#DF-FC02-94ER40818 and by the Deutsche Forschungsgemeinschaft (DFG) under contract We 1254/3-1.

${ }^{\dagger}$ Heisenberg Fellow 
functional (3). Details of this approach and the computation of static nucleon properties are extensively discussed in the literature [2].

\section{REMARKS ON REGULARIZATION}

DIS off the nucleon is parameterized by the hadronic tensor $W^{\mu \nu}(q)$ with $q$ being the momentum transmitted to the nucleon. $W^{\mu \nu}(q)$ is obtained from the nucleon matrix element of the commutator $\left[J^{\mu}(\xi), J^{\nu}(0)\right]$. In the NJL model the current is conveniently given as $J^{\mu}=\bar{q} \mathcal{Q} \gamma^{\mu} q$, with $\mathcal{Q}$ being the quark charge matrix. In the context of functional bosonization it is more appropriate to start from the forward virtual Compton amplitude,

$T^{\mu \nu}(q)=\int d^{4} x \mathrm{e}^{i q \cdot \xi}\left\langle N\left|T\left(J^{\mu}(\xi) J^{\nu}(0)\right)\right| N\right\rangle$,

since the time-ordered product is unambiguously extracted from the regularized action

$T\left(J^{\mu}(\xi) J^{\nu}(0)\right)=\left.\frac{\delta^{2}}{\delta a_{\mu}(\xi) \delta a_{\nu}(0)} \operatorname{Tr}_{\Lambda} \log \left[i \not \partial-\left(S+i \gamma_{5} P\right)+\mathcal{Q} \not \iota\right]\right|_{a_{\mu}=0}$.

In this way the regularization of the structure functions is consistently implemented at the level of the defining action. The hadronic tensor is then obtained from the cut,

$W^{\mu \nu}(q)=\frac{1}{2 \pi} \Im\left(T^{\mu \nu}(q)\right)$.

In order to extract the leading twist piece of the structure functions, $W^{\mu \nu}(q)$ in studied in the Bjorken limit: $q^{2} \rightarrow-\infty$ with $x=-q^{2} / P \cdot q$ fixed. Here $P$ denotes the nucleon momentum. In this limit the leading order contribution in $1 / N_{C}$ to $W^{\mu \nu}(q)$ becomes [3]

$$
\begin{aligned}
& W^{\mu \nu}(q)=i \frac{N_{C}}{4} \int \frac{d \omega}{2 \pi} \sum_{\alpha} \int d^{3} \xi \int \frac{d \lambda}{2 \pi} \mathrm{e}^{i M x \lambda} \\
& \times\left\{\left[\Psi_{\alpha}^{\dagger}(\vec{\xi}) \mathcal{Q}^{2} \gamma^{\mu} \not h \gamma^{\nu} \beta \Psi_{\alpha}\left(\vec{\xi}+\lambda \hat{e}_{3}\right) \mathrm{e}^{-i \lambda \omega}-\Psi_{\alpha}^{\dagger}(\vec{\xi}) \mathcal{Q}^{2} \beta \gamma^{\nu} \not h \gamma^{\mu} \Psi_{\alpha}\left(\vec{\xi}+\lambda \hat{e}_{3}\right) \mathrm{e}^{i \lambda \omega}\right] f_{\alpha}^{(-)}(\omega)_{\text {pole }}\right. \\
& \left.+\left[\Psi_{\alpha}^{\dagger}(\vec{\xi}) \mathcal{Q}^{2} \beta \gamma^{\mu} \not h \gamma^{\nu} \Psi_{\alpha}\left(\vec{\xi}+\lambda \hat{e}_{3}\right) \mathrm{e}^{-i \lambda \omega}-\Psi_{\alpha}^{\dagger}(\vec{\xi}) \mathcal{Q}^{2} \gamma^{\nu} \not h \gamma^{\mu} \beta \Psi_{\alpha}\left(\vec{\xi}+\lambda \hat{e}_{3}\right) \mathrm{e}^{i \lambda \omega}\right] f_{\alpha}^{(+)}(\omega)_{\text {pole }}\right\},
\end{aligned}
$$

with the Pauli-Villars regularized spectral functions,

$f_{\alpha}^{( \pm)}(\omega)=\sum_{i} c_{i} \frac{\omega \pm \epsilon_{\alpha}}{-\omega^{2}+\epsilon_{\alpha}^{2}+\Lambda_{i}^{2}-i \eta} \pm \frac{\omega \pm \epsilon_{\alpha}}{-\omega^{2}+\epsilon_{\alpha}^{2}-i \eta}$

and $\mathrm{n}^{\mu}=(1,0,0,1)$ being a light-cone vector. As there are poles for both positive and negative $\omega$, the meaning of forward and backward moving quarks becomes ambiguous and a description of $W_{\mu \nu}$ in terms of quark distributions seems impossible.

\section{NUMERICAL RESULTS}

Unfortunately no numerical results are currently available for the structure functions as projected from the consistently regularized hadronic tensor (1). Therefore the presentation is limited to the valence quark approximation taking into account only the 
contribution of the distinct valence level. Two observations make this a reliable approximation: (1) This level does not undergo regularization and hence the subtleties mentioned above are avoided. (2) Although the polarized vacuum is mandatory to provide a soliton solution, its contribution to static properties is small or negligible [2]. Sum rules relate them to structure functions, thus it is suggestive that the structure functions are also saturated by the valence quark contribution. For more details the reader is referred to the research papers [ [4, 5, 6] and similar studies by other groups [0, 8].

\subsection{Unpolarized Structure Functions}

The unpolarized structure functions are obtained from the symmetric combination $W_{\mu \nu}+W_{\nu \mu}$. The result for the structure function which enters the Gottfried sum rule of $e-N$ scattering is shown in fig. 1. The boost to the infinite momentum frame [ 9] mitigates the effects of omitting the dynamical response of the soliton to the infinite momentum transfer and provides proper support for the structure functions. A DGLAP evolution [ 10] determines the low energy scale $Q_{0}^{2} \approx 0.4 \mathrm{GeV}^{2}$ at which the model supposedly approximates QCD. The model reproduces the gross features of the experimental data although an even better agreement can be gained by further reducing $Q_{0}^{2}$ which, however, would make the DGLAP program unrealistic. Depending on the model parameter $m$, the Gottfried sum rule, $S_{G}=\int d x\left(F_{2}^{\mathrm{ep}}-F_{2}^{\mathrm{en}}\right) / x$ is found to be $0.26-0.29$ which exhibits the desired deviation from the historic value $(1 / 3)$ demanded empirically, $0.235 \pm 0.026$ [ 11.

Fig. 1: The unpolarized structure function entering the Gottfried sum rule. RF: rest frame, IMF: boosted to the infinite momentum frame, LO: leading order QCD evolution to $Q^{2}=$ $4 \mathrm{GeV}^{2}$. Data are from the NMC [11].

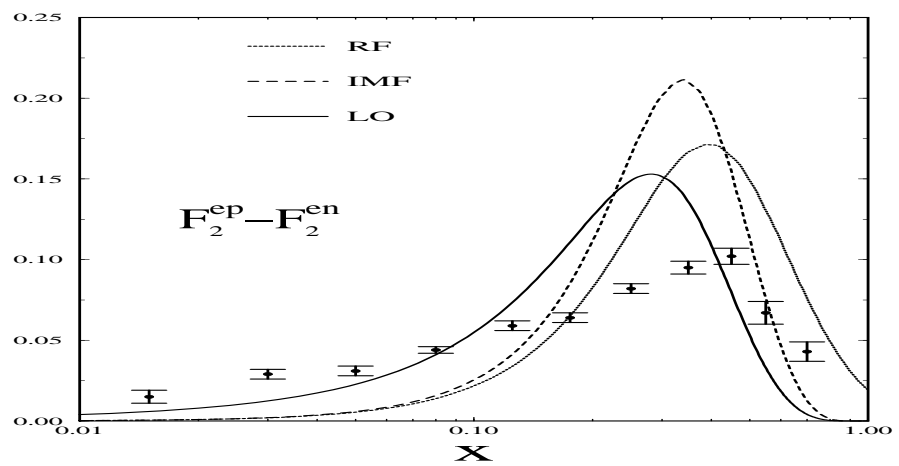

\subsection{Spin Structure Functions}

The spin - polarized structure functions $g_{1}$ and $g_{2}$ are obtained from the anti-symmetric combination $W_{\mu \nu}-W_{\nu \mu}$. While the former is related to the proton spin puzzle, the latter sheds some light on higher twist effects [12]. Typical results are shown in fig. 2 and are compared to data from SLAC [13]. Apparently a reasonable agreement is obtained. 
Fig. 2: Model predictions for the polarized nucleon structure functions $g_{1}$ (left panel) and $g_{2}$ (right panel). Data are from SLAC [13].
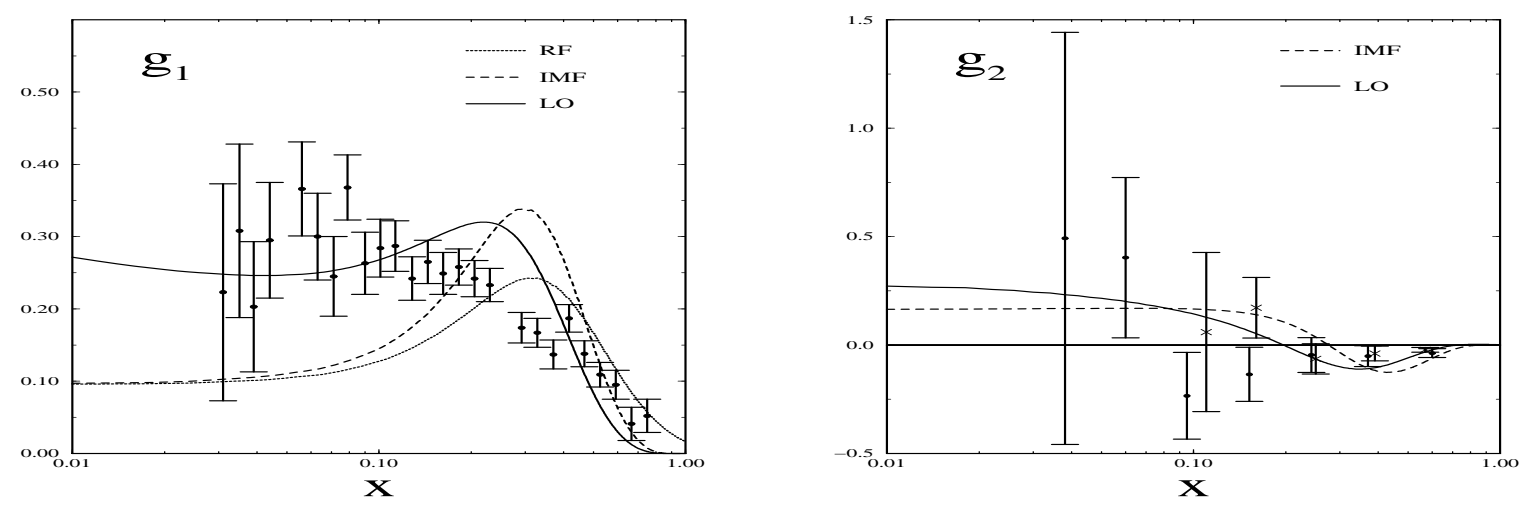

\subsection{Generalization to Flavor $\mathrm{SU}(3)$}

The model can straightforwardly be generalized to also include strange quarks through the collective coordinate quantization [ [6]. In a flavor symmetric formulation one would expect sizable strange quark contributions to all nucleon properties. Fortunately flavor symmetry breaking effects can be included, thereby considerably reducing the strange quark contribution to $g_{1}, c f$. fig. 3 . This is also reflected by the small difference between the two and three flavor model calculation for $g_{1}$ of the proton.

Fig. 3: Left panel: Strangeness contribution to $g_{1}$. Right panel: Comparison of the two and three flavor model calculation of $g_{1}$ of the proton. Data are from ref [ 13].
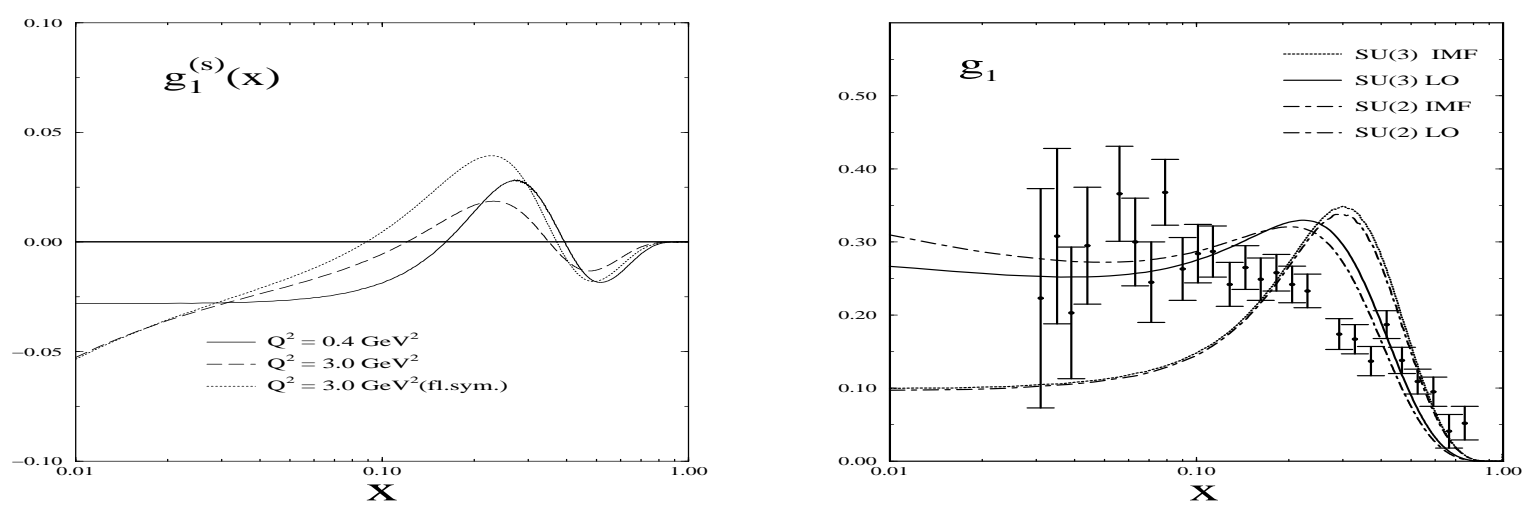

\section{CONCLUSIONS}

Nucleon structure functions can be computed from a chiral soliton model which describes baryons as lumps of mesons. All relevant information is contained in the hadronic tensor which can be computed from the symmetry currents of the model. Thus the identification of degrees of freedom with those of QCD, which seems impossible after regularization, is unnecessary. Reasonable agreement with experimental data is obtained in the valence quark approximation which leaves aside the technical subtleties of regularization. 


\section{ACKNOWLEDGMENTS}

It is my pleasure to thank the organizers for providing a very pleasant and memorable symposium. The material presented stems from collaborations with L. Gamberg, H. Reinhardt, O. Schröder and E. Ruiz Arriola whose contributions are gratefully acknowledged.

\section{REFERENCES}

[1] Y. Nambu and G. Jona-Lasinio, Phys. Rev. 122 (1961) 345; 124 (1961) 246,

D. Ebert and H. Reinhardt, Nucl. Phys. B271 (1986) 188.

[2] R. Alkofer, H. Reinhardt and H. Weigel, Phys. Rep. 265 (1996) 139,

C. V. Christov et al., Prog. Part. Nucl. Phys. 37 (1996) 91.

[3] H. Weigel, E. Ruiz Arriola and L. Gamberg, in preparation.

[4] H. Weigel, L. Gamberg and H. Reinhardt, Mod. Phys. Lett. A11 (1996) 3021, Phys. Lett. B399 (1997) 28.

[5] H. Weigel, L. Gamberg and H. Reinhardt, Phys. Rev. D55 (1997) 6910,

L. Gamberg, H. Reinhardt and H. Weigel, Phys. Rev. D58 (1998) 054014.

[6] O. Schröder, H. Reinhardt and H. Weigel, Phys. Lett. B439 (1998) 398, hep-ph/9902322.

[7] D. I. Diakonov et al., Nucl. Phys. B480 (1996) 341, Phys. Rev. D56 (1997) 4069.

[8] M. Wakamatsu and T. Kubota, Phys. Rev. D57 (1998) 5755, hep-ph/9809443.

[9] L. Gamberg, H. Reinhardt and H. Weigel, Int. J. Mod. Phys. A13 (1998) 5519.

[10] V. N. Gribov and L. N. Lipatov, Sov. J. Nucl. Phys. 15 (1972) 438,

Y. L. Dokshitzer, Sov. Phys. JETP 46 (1977) 461,

G. Altarelli and G. Parisi, Nucl. Phys. B126 (1977) 298.

[11] M. Arneodo et al. (NMC), Phys. Rev. D50 (1994) R1.

[12] R. L. Jaffe, "Spin, Twist and Hadron Structure..." Erice Lectures 1995, hep-ph/9602236.

[13] K. Abe et al., Phys. Rev. Lett. 74 (1995) 346. 